\title{
Histological and Radiographical Evaluation of Deciduous Teeth during Shedding (Human and Experimental Study)
}

\author{
Mai Badreldin Helal* \\ Department of Oral Biology, Faculty of Dentistry, Tanta University, Tanta, Egypt
}

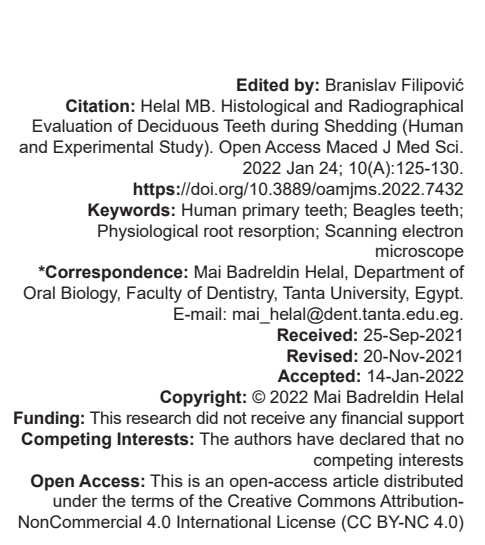

Abstract

AIM: The aim of this study was to evaluate the process of deciduous teeth shedding histologically and radiographically. METHODS: The design of the present study included both human and experimental animals. A total number of twenty human primary teeth, aged 8-10 years, were collected for light microscope and scanning electron microscopy (SEM). Furthermore, ten nameless copies of dental/occlusal X-rays of children aged 9-10 years were used to measure the radicular dentin radiodensity. For the experimental part, 4-month-old beagles were used for histological examination of the process of shedding in situ.

RESULTS: Histologically, the decalcified beagles deciduous teeth specimens showed deep resorption fossae occupied with many odontoclasts together with periodontal ligaments disorganization. Furthermore, SEM examination of human exfoliated teeth revealed variable-sized plentiful resorption lacunae with irregular edges. Interestingly, radiographic examination of the human deciduous teeth at late resorption stage revealed significant decrease in radicular dentin radiodensity.

CONCLUSION: Shedding is a complex physiological process that involves intermittent resorption of deciduous teeth supporting tissues together with significant decrease in root dentin radiodensity at late root resorption stage in comparison to other various stages of root resorption.

\section{Introduction}

Human and diphyodont animals have two sets of dentition; deciduous and permanent teeth. The necessity for two dentitions exists, since teeth, once formed, cannot increase in size [1]. Thus, a second dentition, consisting of larger and more teeth is required to cope with jaw growth and to bear adult masticatory forces [2]. Regarding beagles' deciduous teeth, they have two sets of teeth in the form of 28 deciduous teeth being exfoliated and replaced by a 42-permanent set [3].

Primary teeth exfoliation or shedding is a physiological process, that is characterized by the elimination of deciduous dentition after resorption of their roots and their supporting tissues. Histologically, odontoclasts appear on the apical surface of deciduous teeth and tend to initiate resorption through the removal of apatite crystals and organic matrix degradation of both root cementum and dentin [4]. Many theories explained the factors that influence the shedding process. One of them is the pressure applied by the growing and erupting permanent tooth germ which is responsible for the initiation of odontoclasts differentiation. Furthermore, mechanical-occlusal trauma and inflammatory processes were proved to play an important role in the shedding process [5].
Interestingly, deciduous teeth exfoliation is caused by intermittent resorption of their roots prior to the eruption of their permanent successors [6]. Although, this unceasing intermittent root resorption that occurred during shedding might affect dentin mineral content and radiodensity, little or no information has been conducted to describe the affection of root dentin radiodensity during different stages of root resorption [7].

Consequently, the present study aimed to examine the process of shedding using histological (light and scanning electron microscopic (SEM) examination) together with radiographical evaluation of the radicular dentin radiodensity during different root resorption stages.

\section{Materials and Methods}

The design of the current study included both human and experimental animals. A total number of 20 human primary teeth, age range 8-10 years, were collected from the Pediatric Dental Clinics at Faculty of Dentistry, Tanta University. Teeth were extracted before shedding. Sample size was calculated using StateCalc 
under Epi info software with a two-sided confidence level $95 \%$ and a power $85 \%$. Considering we have used 20 specimens divided for SEM and light microscope (LM) examination. Teeth collection was conducted in accordance with the guidelines on the human research approved by the Ethical Committee at Faculty of Dentistry Tanta University, Egypt. Furthermore, ten nameless copies of dental/occlusal X-rays of children deciduous teeth were used for dentin radiodensity evaluation. The X-rays were obtained after taking the patients permission.

For the experimental part, 4-month-old purebred beagles were used for histological examination of the process of shedding in situ. Similar animals were used in previous studies concerning shedding [8]. Furthermore, dental X-rays were done to beagles jaws to confirm their mixed dentition and the condition of their primary premolars. Study protocols and animal handling were approved by the Ethical Committee at Faculty of Dentistry Tanta University, Egypt.

\section{Methods of investigations for exfoliated human teeth}

\section{Light microscopic examination}

Ten exfoliated human teeth including both incisors and molars were dissected and immersed in $10 \%$ buffered paraformaldehyde for $48 \mathrm{~h}$. The teeth were decalcified in $10 \%$ ethylenediaminetetraacetic acid (EDTA) solution for 6 weeks. Then, teeth were dehydrated, embedded in paraffin, sectioned mesiodistally at $5 \mu \mathrm{m}$ thickness then stained with hematoxylin and eosin [9].

\section{SEM examination}

Ten exfoliated human teeth were assigned into two groups according to their types; anterior and posterior and processed for SEM examination.

All deciduous teeth were fixed in a solution containing $2.5 \%$ glutaraldehyde and $2 \%$ paraformaldehyde in $0.1 \mathrm{M}$ phosphate buffer $(\mathrm{pH} 7.2-$ 7.4) for $24-48 \mathrm{~h}$ at $4^{\circ} \mathrm{C}$. Teeth were then rinsed with distilled water and immersed in a $0.5 \%$ sodium hypochlorite solution for $1 \mathrm{~h}$ at room temperature. After that, they were rinsed with distilled water for $12 \mathrm{~h}$ at $4^{\circ} \mathrm{C}$ and post-fixed in $1 \%$ osmium tetroxide for $2 \mathrm{~h}$ at $4^{\circ} \mathrm{C}$. Specimens were then dehydrated with ascending graded ethanol $(70 \%, 80 \%, 90 \%, 95 \%$, and $100 \%$ ethanol) and dried with $\mathrm{CO}_{2}$ liquid in a critical point dryer. They were mounted on metal stubs, coated with gold/ palladium using an ion sputter [10]. Then, the processed exfoliated human primary teeth were examined using SEM (JSM 5600LV, Jeol, Tokyo, Japan) in electron microscope unit of the Faculty of Science, Alexandria University.
Measurement of the radicular dentin

radiodensity

Ten nameless copies of dental/occlusal x-rays of children deciduous teeth at various stages of root resorption taken after their parents permission were incorporated in this analysis.

Standardized photographs were taken at the same time for quantitative evaluation of dentin radiodensity. Images were captured with the exposure time $(0.8 \mathrm{~s})$ and the target-sensor distance $(60 \mathrm{~cm})$ and were standardized for all the radiographs. The X-ray beam direction was standardized to avoid any possible influence of these factors on the radiodensity of the images. Then, the dental/occlusal X-rays were divided into two groups according to root resorption stage; early stage of root resorption was defined as resorption of not more than one-third of the root length, whereas the late stage of root resorption was defined as resorption of more than one-third of the root length.

In order to measure the degree of root resorption for each tooth, a definite step was followed according to [11]. First, the distance between the enamel-cement junction and the deepest point of root resorption was measured using an electronic millimeter caliper. The resultant measurement was then divided by the pre-resorption total root length for that specific tooth type.

The radicular dentin radiodensity measurement was performed on the images using the Image $J$ analysis software (Image J 1.42q, Wayne Rasband, USA). Five different fields were examined for the mean intensity gray value in five different $X$-rays. The optical density (OD) values were identified according to [12] using this formula $\mathrm{OD}=\log$ (maximum intensity/mean intensity), where max intensity $=255$ for 8 -bit image.

\section{Methods of investigations for experimental study for shedding}

The deciduous premolars obtained from beagles were dissected and immersed in $10 \%$ buffered paraformaldehyde for $48 \mathrm{~h}$. The specimens were decalcified in $10 \%$ EDTA solution for 8 weeks. Then, specimens were dehydrated, embedded in paraffin, sectioned mesiodistally at $5 \mu \mathrm{m}$ thickness then stained with hematoxylin and eosin.

\section{Statistical analysis}

The quantitative data collected from the measurement of dentin radiodensity were tabulated and statistically analyzed using CO-STAT analysis (version 6.4). Numerical variables were expressed by descriptive statistics as mean, standard deviation, and range. One-way ANOVA and post hoc test (Tukey-test) were used to compare quantitative data between early and late resorption groups. 


\section{Results}

\section{Exfoliated human teeth}

\section{Light microscopic results}

In the current study, light microscopic examination of the exfoliated human teeth revealed numerous, large resorption lacunae (Figure 1b). The appearance of the secondary dentin was observed in the incisal portion of the exfoliated primary incisors (Figure 1a).

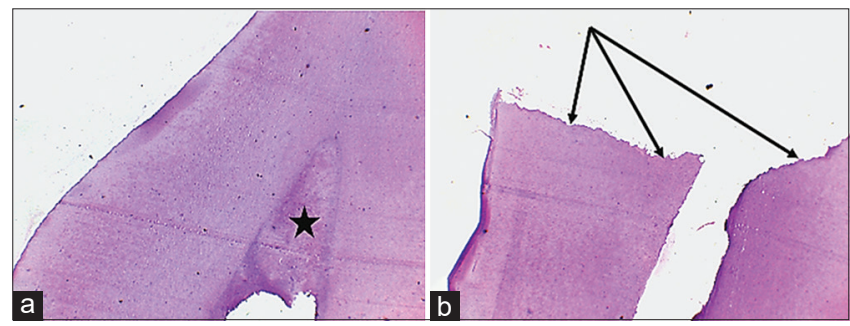

Figure 1: Light micrographs of exfoliated human deciduous teeth, (a) The exfoliated tooth shows a thick layer of secondary dentin (star) that has been deposited in the incisal area. (b) The root surface displays numerous resorption lacunae created by odontoclasts. The pulp tissue detachment occurred artefactually during specimen preparation

\section{SEM results}

SEM for exfoliated human anterior and posterior teeth

In the present study, the exfoliated incisors revealed extensive loss of cementum from the entire lingual aspect of their roots creating a ragged appearance of the root surface, with complete or partial cementum resorption (Figure 2a and b). Remarkably, both external and internal root resorption were also reported in some exfoliated incisors. The internally resorbed pulpal surface reveals odontoclasts in their lacunae (Figure 2c and d). Similarly, SEM examination of root resorption surface of deciduous molars revealed loss of normal smooth topography of the root surface with irregularly shaped resorption lacunae that had a circular or polygonal shape (Figure $3 a$ and $b$ ).

\section{Radiographic examination for exfoliated} human teeth

In general, radiographic records of the human deciduous teeth undergoing shedding revealed different shedding patterns. In case of the deciduous anterior teeth, resorption was detected on the lingual surfaces of their roots. This occurs as their successors develop lingually and erupt in an occlusal and vestibular direction (Figure $4 \mathrm{a}$ and $\mathrm{b}$ ). On the other hand, the tooth germs of the developing premolars were detected between the divergent flared roots of the deciduous molar (Figure 5).

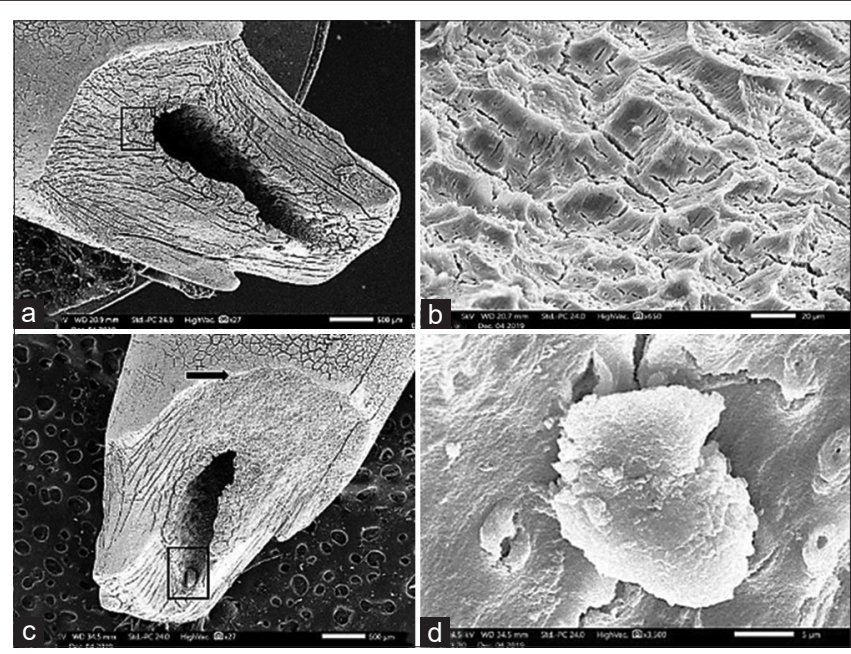

Figure 2: (a-d) Scanning electron micrograph of the human exfoliated primary upper central incisor. (a) Exhibits external lingual root resorption (squared area). (b) Higher magnification displays eroded root surface with numerous resorption lacunae created by odontoclasts. Notice, complete resorption of cementum with exposed dentin. (c) Image displays both external (thick black arrow) and internal root resorption. Notice, odontoclast is present in its lacunae on the inner pulp surface (squared area). (d) Higher magnification of the previous squared area shows odontoclast in its lacunae
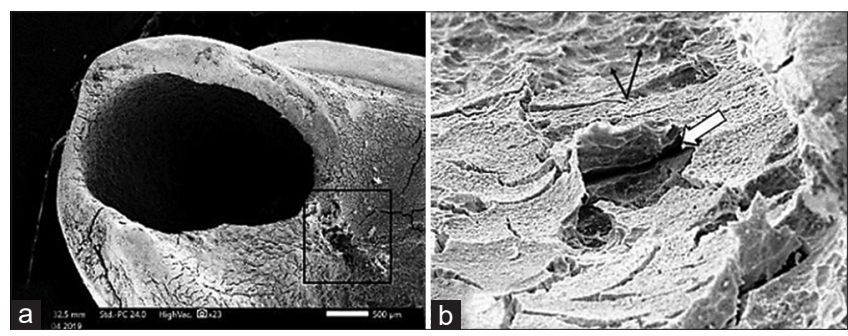

Figure 3: $(a$ and $b)$ Scanning electron micrograph of the root surface of an exfoliated human deciduous first molar tooth. (a) The apical and inter radicular regions of the root reveal external root resorption (squared area). (b) higher magnification of the squared area displays shallow (black arrows) and deep (white arrow) resorption lacunae on the root surface
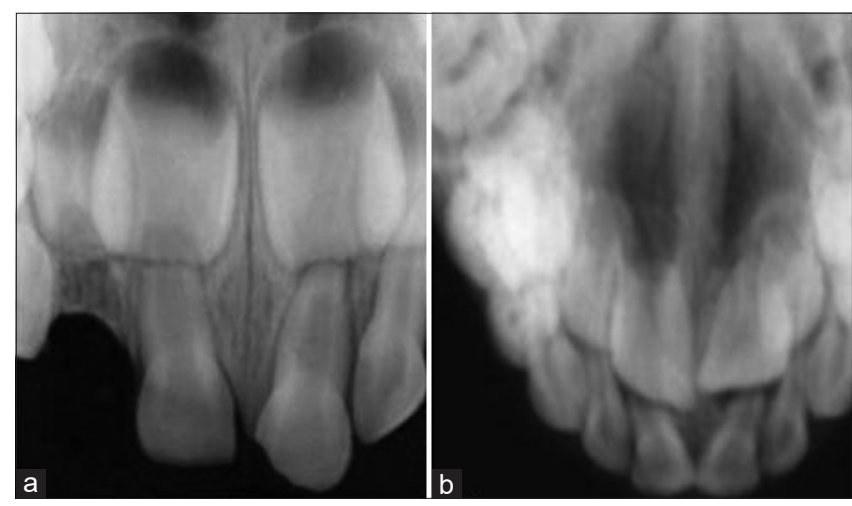

Figure 4: (a) Radiographic image of exfoliating deciduous maxillary incisor at the early root resorption stage. Notice, the permanent successors are lingually located. (b) Radiographic image of deciduous maxillary incisors that reveal late root resorption stage

Regarding dentin radiodensity, a significant difference in dentin radiodensity was observed between early and late root resorption stages (Table 1 


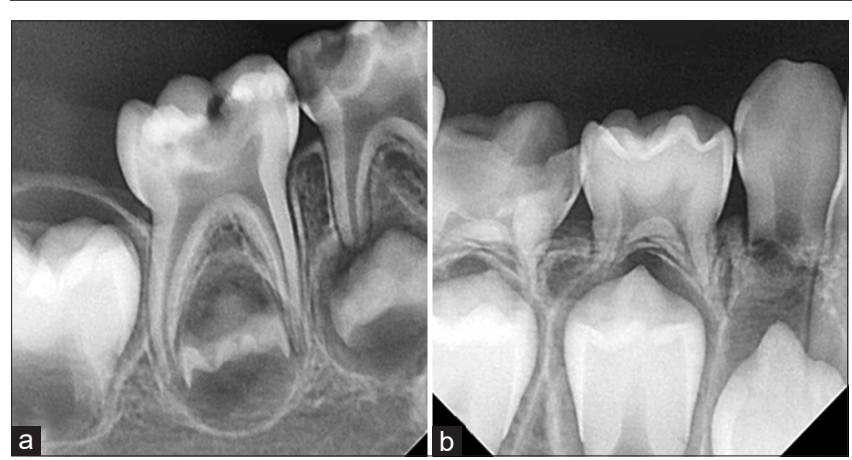

Figure 5: ( $a$ and b) Radiographic image of part of child mandible shows the location of the permanent successors below the divergent roots of the deciduous molars. (a) Radiographic image of exfoliating deciduous mandibular molar at the early root resorption stage. (b) Radiographic image of deciduous mandibular molars that display late root resorption stage

and Figure 6). As radicular dentin in roots with late resorption stage displayed a significant decrease in dentin radiodensity.

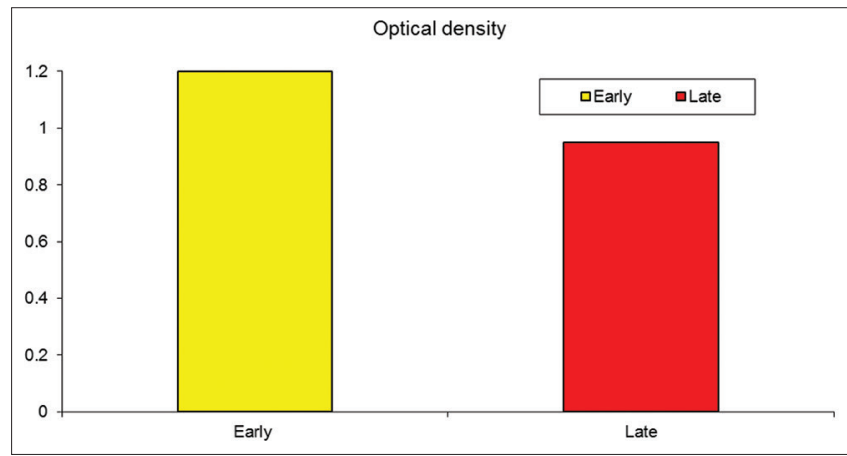

Figure 6: Histogram shows comparison between early and late resorption stages

\section{Experimental study for shedding} mandible

Radiographic examination for beagles

Radiographic image of the beagles mandible disclosed shedding of deciduous premolar. The developing permanent premolars were located between the divergent roots of their predecessors.

\section{Light microscopic examination}

Histological examination of mesiodistal sections of exfoliating dog premolars reveals alteration of their root cementum, periodontal ligament, pulp, and bone.

Table 1: Data was presented as range, means \pm SD ( $n=25 /$ group) comparing between early and late resorption stages. *Statistically significant

\begin{tabular}{lll}
\hline Optical density & Early resorption stage & Late resorption stage \\
\hline Range & $1.08-1.98$ & $0.53-1.28$ \\
Mean \pm SD & $1.20 \pm 0.19$ & $0.95 \pm 0.19$ \\
t-test & 4.528 & \\
p-value & $0.001^{\star}$ & \\
\hline
\end{tabular}

Cementum, periodontal ligament, and pulp response

The current study revealed physiological root resorption of the deciduous teeth. There are numerous, large multinucleated odontoclasts residing at the Howship's lacunae. The odontoclasts resorbed hard dental tissues, including cementum and dentin on the external root portion. The resorption areas were demonstrated as deep round wave-like resorption lacunae (Figure 7a and b). Remarkably, the appearance of the repairing cellular cementum was clearly observed covering the resorbed regions (Figure 7c). Furthermore, the periodontal ligaments displayed disruption and disorganization in the areas of root resorption (Figure $7 a$ and d). On the other hand, the pulp of the deciduous teeth appeared almost normal, with intact odontoblastic zone.

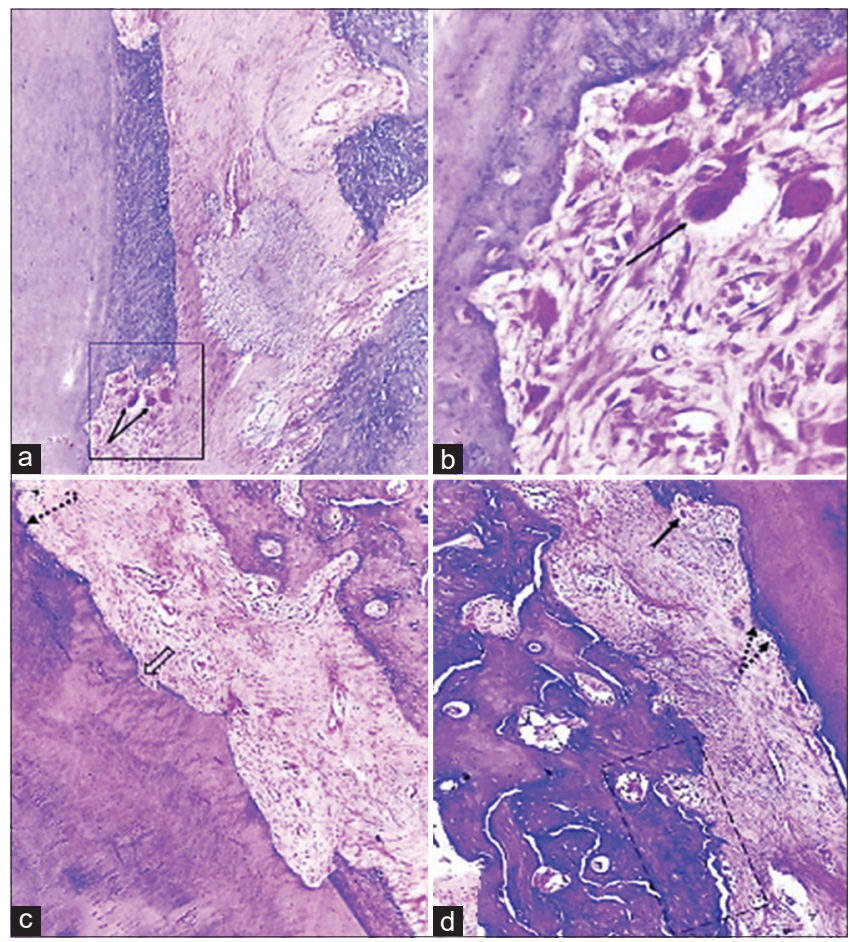

Figure 7: (a-d) Light micrographs of beagles deciduous lower premolar. (a) Image displays large resorption areas that are occurring at the apical root region. The cementum shows numerous odontoclasts in their lacunae (black arrows) in association with areas of periodontal ligament disorganization (white arrow). (b) Higher magnification of the square area in A shows numerous multinucleated odontoclasts in resorption lacunae (black arrow). (c) The apical root portion shows extensive cementum and dentin resorption (open arrow) together with deposition of cementum-like tissue in the resorption lacuna (dashed black arrows). (d) The root surface shows resorption lacunae containing odontoclasts (black arrow) along with periodontal ligament disorganization and alveolar bone resorption (dashed rectangle) (H\&E orig. mag, $A \times 100, B \times 1000, C, D \times 100$ )

\section{Bone response}

In the current study, light microscopic examination of beagles deciduous premolar teeth histological sections revealed resorption of bone with multiple osteoclasts in their resorption lacunae (Figure 7d). 


\section{Discussion}

Despite previous researchers described the histological changes that occurred during deciduous teeth exfoliation [13], no studies have evaluated the variation in radicular dentin radiodensity of the teeth undergoing shedding. The current study aimed to evaluate the histological and radiographical changes that occurred during shedding process in human and beagles.

The use of experimental animals besides human exfoliated teeth in our research to provide accurate understanding of the histological events that occur during shedding process. As beagles histological sections facilitate examination of the primary teeth supporting tissues [14].

This discussion will focus on the explanation of the histological (LM and SEM) and radiographical changes that occurred in the exfoliated teeth. The LM examination of both human and beagles exfoliated teeth confirmed the observation made previously by other investigators [15], who reported that shedding process is initiated by deciduous teeth root resorption. There are many theories that had explained the process of root resorption commencement. First, the local factors as an etiological factor that initiate shedding of the deciduous teeth. Researchers reported that the pressure caused by the underlying developing permanent teeth germs resulted in activation of odontoclasts and ultimately teeth exfoliation [7]. Since it was verified that the pattern of human deciduous teeth resorption is dictated by the pressure and position of their successional tooth. To evaluate this theory, Linsuwanont et al. [16] studied the effects of removing the permanent tooth germs in beagles. They found that resorption of the roots of the deciduous predecessors was delayed than physiological resorption. The later finding was also in agreement with the clinical observation made by Kashyap et al. [17] who reported that a retardation occurred in the shedding of a deciduous tooth when its successor is congenitally absent or misplaced in an abnormal position.

Second, besides the previously reported local factors causing the dental hard tissue resorption, genetic factors that considered shedding process as a pre-programmed event had been reported as an etiological factor. On the molecular level root resorption was reported to be initiated by stimulatory molecules secreted by the underlying permanent teeth follicle and reduced enamel epithelium [15]. In addition, the primary root resorption process was reported to be regulated in a manner similar to bone remodeling, concerning the same receptor-ligand system known as receptor activator of nuclear factor-kappa B/RANK Ligand (RANK/RANKL) [18]. Furthermore, HarokopakisHajishengallis, [19] dictated the evenness between the left and right side of the mouth regarding the exfoliation timing of the primary teeth and of the emergence of the permanent successors suggested that shedding of the primary teeth may be a programmed event.

Interestingly, the involvement of the periodontal ligament that was observed in our experimental part has been confirmed to have a definite role in the process of root resorption of the deciduous teeth [20]. Precisely, the removal of the collagen fibers of the PDL is considered a main step in the initiation of the resorption process. In the current study, the histological sections of beagles teeth periodontal ligament showed signs of insults in the form of disorganization and hyalinization. Noteworthy, it was reported that degradation of periodontal ligament precedes root resorption as a result of heavier masticatory forces that are applied on the primary tooth periodontal ligament [21]. On the molecular level, Song et al. [22] declared that the extracellular matrix-degrading enzymes, including matrix metalloproteinases and collagenase were found to be increased in the PDL tissues obtained from deciduous teeth undergoing root resorption.

Unexceptionally, the histological examination of dental pulp of the beagles exfoliated teeth, at this time of resorption revealed normal pulpal tissues, and dental pulp did not seem to participate in the resorption process. This was in agreement with Sahara et al. [23], who reported that the odontoclasts existence inside the pulp occurred only at the late stage of root resorption.

The use of SEM in studying root resorption in human exfoliated teeth has an advantage in giving an accurate examination of the resorbed surfaces and allowed observation of the morphology of mineralized tissues in multiple planes which could not be obtained by histological examination only. In the present study, the primary incisors and molar teeth examined with SEM were in different stages of root resorption but most of them had lost more than one thirds to twothirds of their root length prior to exfoliation. A notable feature was observed clinically in our exfoliated primary molars, was that the resorption was of varying extent in different roots of the same molar. We need to recall that resorption of primary teeth is not solely dependent on the pressure of erupting permanent teeth on the primary predecessor, it also depends on factors originating from the succeeding permanent follicle. Thus, molar roots located closer to the follicle showed a greater degree of resorption than others [19].

Regarding the radiographic obtained data that reported a significant decrease in dentin radiodensity during the late resorption stage. The later observation was in agreement with Matsuda, [24], who studied the distribution of organic mass at sites of active bone resorption by means of ultra-soft X-rays. He reported a band of increased X-ray absorption which was closely associated with the most superficial layer of the bone matrix and assumed that this alteration in radiodensity might be due to the zone of decreased mineral density. 


\section{Conclusion}

Our study portrayed that shedding process is a timed physiological process that involves both dental hard tissue resorption together with alteration in radicular dentin radiodensity.

\section{References}

1. Nanci ABT-TCOH. Physiologic Tooth Movement: Eruption and Shedding. 8th ed., Ch. 10. Massachusetts, United States: Mosby; 2013. p. 233-52. https://doi.org/10.1016/ B978-0-323-07846-7.00010-0

2. Sahara N. Cellular events at the onset of physiological root resorption in rabbit deciduous teeth. Anat Rec. 2001;264(4):387-96. https://doi.org/10.1002/ar.10017 PMid:11745094

3. Sahara N, Okafuji N, Toyoki A, Suzuki I, Deguchi T, Suzuki K. Odontoclastic resorption at the pulpal surface of coronal dentin prior to the shedding of human deciduous teeth. Arch Histol Cytol. 1992;55(3):273-85. https://doi.org/10.1679/aohc.55.273 PMid:1419277

4. Sahara N, Okafuji N, Toyoki A, Ashizawa $Y$, Yagasaki $H$ Deguchi T, et al. A histological study of the exfoliation of human deciduous teeth. J Dent Res. 1993;72(3):634-40. https://doi.org $/ 10.1177 / 00220345930720031401$

PMid:8450123

5. Sahara N, Ashizawa Y, Nakamura K, Deguchi T, Suzuki K. Ultrastructural features of odontoclasts that resorb enamel in human deciduous teeth prior to shedding. Anat Rec. 1998;252(2):215-28. https://doi.org/10.1002/ (SICI)1097-0185(199810)252:2<215:AID-AR7>3.0.CO;2-1 PMid:9776076

6. Yildirim S, Yapar M, Sermet U, Sener K, Kubar A. The role of dental pulp cells in resorption of deciduous teeth. Oral Surg Oral Med Oral Pathol Oral Radiol Endodontol. 2008;105(1):113-20. https://doi.org/10.1016/j.tripleo.2007.06.026 PMid:17942342

7. Sahara N, Ozawa H. Cementum-like tissue deposition on the resorbed enamel surface of human deciduous teeth prior to shedding. Anat Rec Part A Discov Mol Cell Evol Biol. 2004;279(2):779-91. https://doi.org/10.1002/ar.a.20069 PMid: 15278949

8. Cahill DR. Histological changes in the bony crypt and gubernacular canal of erupting permanent premolars during deciduous premolar exfoliation in beagles. J Dent Res. 1974;53(4):786-91. https://doi.org/10.1177/00220345740530040301 PMid:4526370

9. Alturkistani HA, Tashkandi FM, Mohammedsaleh ZM Histological stains: A literature review and case study. Glob J Health Sci. 2015;8(3):72-9. https://doi.org/10.5539/gjhs. v8n3p72

PMid:26493433

10. Stutzman PE, Clifton JR. Specimen Preparation for Scanning Electron Microscopy. Vol. 21. In: Proceedings of the International Conference on Cement Microscopy; 1999. p. 10-22.

11. Francini $E$, Mancini $G$, Vichi $M$, Tollaro $I$, Romagnoli $P$. Microscopical aspects of root resorption of human deciduous teeth. Ital J Anat Embryol. 1992;97(3):189-201.

PMid:1285684

12. Brookes SJ. Using ImageJ (Fiji) to analyze and present X-ray CT images of enamel. In: Odontogenesis. Berlin: Springer; 2019. p. 267-91.

13. Eronat $\mathrm{C}$, Eronat N, Aktug M. Histological investigation of physiologically resorbing primary teeth using Ag-NOR staining method. Int J Paediatr Dent. 2002;12(3):207-14. https://doi. org/10.1046/j.1365-263x.2002.00337.x PMid:12028313

14. Pound P, Ebrahim S, Sandercock P, Bracken MB, Roberts I. Where is the evidence that animal research benefits humans? BMJ. 2004;328(7438):514-7. https://doi.org/10.1136/bmj.328.7438.514 PMid:14988196

15. Hammarström L, Lindskog S. Factors regulating and modifying dental root resorption. Proc Finn Dent Soc. 1992;88(Suppl 1):115-23.

\section{PMid:1508866}

16. Linsuwanont $B$, Takagi $Y$, Ohya $\mathrm{K}$, Shimokawa $\mathrm{H}$. Expression of matrix metalloproteinase-9 mRNA and protein during deciduous tooth resorption in bovine odontoclasts. Bone. 2002;31(4):4728. https://doi.org/10.1016/s8756-3282(02)00856-6 PMid:12398942

17. Kashyap RR, Kashyap RS, Kini R, Naik V. Prevalence of hyperdontia in nonsyndromic South Indian population: An institutional analysis. Indian J Dent. 2015;6(3):135-8. https://doi. org/10.4103/0975-962X.163044

PMid:26392730

18. Ogasawara T, Yoshimine $\mathrm{Y}$, Kiyoshima T, Kobayashi I, Matsuo K, Akamine A, et al. In situ expression of RANKL, RANK, osteoprotegerin and cytokines in osteoclasts of rat periodontal tissue. J Periodontal Res. 2004;39(1):42-9. https:// doi.org/10.1111/j.1600-0765.2004.00699.x

19. Harokopakis-Hajishengallis E. Physiologic root resorption in primary teeth: molecular and histological events. J Oral Sci. 2007;49(1):1-12. https://doi.org/10.2334/josnusd.49.1 PMid: 17429176

20. Sasaki T, Shimizu T, Watanabe C, Hiyoshi Y. Cellular roles in physiological root resorption of deciduous teeth in the cat J Dent Res. 1990;69(1):67-74. https://doi.org/10.1177/0022034 5900690011101

PMid:2303598

21. Otsuka K, Pitaru S, Overall CM, Aubin JE, Sodek J. Biochemical comparison of fibroblast populations from different periodontal tissues: Characterization of matrix protein and collagenolytic enzyme synthesis. Biochem Cell Biol. 1988;66(3):167-76. https://doi.org/10.1139/o88-023 PMid:2838055

22. Song JS, Hwang DH, Kim SO, Jeon M, Choi BJ, Jung HS, et al Comparative gene expression analysis of the human periodontal ligament in deciduous and permanent teeth. PLoS One. 2013;8(4):e61231. https://doi.org/10.1371/journal.pone.0061231 PMid:23593441

23. Sahara N, Toyoki A, Ashizawa $\mathrm{Y}$, Deguchi T, Suzuki K. Cytodifferentiation of the odontoclast prior to the shedding of human deciduous teeth: An ultrastructural and cytochemical study. Anat Rec. 1996;244(1):33-49. https://doi.org/10.1002/ (SICI)1097-0185(199601)244:1<33:AID-AR4>3.0.CO;2-G PMid:8838422

24. Matsuda E. Ultrastructural and cytochemical study of the odontoclasts in physiologic root resorption of human deciduous teeth. Microscopy. 1992;41(3):131-40.

PMid:1328451 